\title{
Fourier transform-wavefront reconstruction for the pyramid wavefront sensor
}

\author{
Fernando Quirós-Pacheco ${ }^{1, a}$, Carlos Correia $^{2}$, and Simone Esposito ${ }^{1}$ \\ 1 INAF - Osservatorio Astrofisico di Arcetri, Italy. \\ 2 ONERA, France.
}

Abstract. The application of Fourier-transform reconstruction techniques to the pyramid wavefront sensor has been investigated. A preliminary study based on end-to-end simulations of an adaptive optics system with $\approx 40 \mathrm{x} 40$ subapertures and actuators shows that the performance of the Fourier-transform reconstructor (FTR) is of the same order of magnitude than the one obtained with a conventional matrix-vector multiply (MVM) method.

\section{Introduction}

In this paper we will present a preliminary study on the application of the Fourier Transform Reconstructor (FTR) to the pyramid wavefront sensor. This work is relevant, for instance, for the extreme AO system of EPICS (the planet finder for the E-ELT), in which the pyramid has been indentified as an optimal wavefront sensor for its halo rejection capabilities at small angular separations [1]. It is envisaged that this XAO system will comprise 30000 actuators and subapertures. Clearly, the FTR would be advantageous to cope with such system dimensions.

Fourier-domain reconstruction techniques have been widely studied for the Shack-Hartmann wavefront sensor (SHWFS). Different inverse filters based on simple sensor models (i.e. the Hudgin or the Fried geometries) have been proposed so far [2]. In this work we have studied the applicability of these inverse filters to the pyramid wavefront sensor. We will show that even using these simple models (not tuned for the pyramid sensor) we can achieve a good wavefront reconstruction and correction. The derivation of ad-hoc inverse filters for the pyramid sensor is beyond the scope of this preliminary work.

We have studied the performance of the Fourier reconstruction techniques with numerical simulations. We have considered the case of an 8-m class AO system. The number of subapertures and actuators that will be considered in this study is $\approx 40 \mathrm{x} 40$. This system configuration is similar to the $\mathrm{XAO}$ systems currently under investigation for the VLT.

\section{Wavefront Sensor Signal Models}

Similarly to the SHWFS, the signals of the PWFS also contain information of the gradient of the incoming wavefront. Indeed, following geometrical optics theory, it can be shown that the PWFS signals are a function of the phase derivatives $[3,4]$ :

$$
\begin{aligned}
& S_{x}(x, y)=\frac{2}{\pi} \arcsin \left[\frac{1}{a_{t t}} \frac{\lambda}{2 \pi} \frac{\partial}{\partial x} \varphi(x, y)\right] \\
& S_{y}(x, y)=\frac{2}{\pi} \arcsin \left[\frac{1}{a_{t t}} \frac{\lambda}{2 \pi} \frac{\partial}{\partial y} \varphi(x, y)\right]
\end{aligned}
$$

where $a_{t t}$ denotes the angular amplitude of the circular modulation. More accurate expressions for the PWFS signals can be obtained from diffractive optics theory. Since this treatment is more elaborate,

\footnotetext{
a e-mail: fquiros@arcetri.astro.it
} the original work is properly cited. 
we have decided to start with the simplest models available (namely the Hudgin and the Fried models widely used for the SHWFS) and evaluate how well they can match the response of the pyramid WFS.

Recall that in the Hudgin model, the signals are considered to be the first-order differences of the phase $\varphi(x, y)$, that is:

$$
\begin{aligned}
& S_{x}^{H}\left(x_{n}, y_{n}\right)=\varphi\left(x_{n}, y_{n+1}\right)-\varphi\left(x_{n}, y_{n}\right) \\
& S_{y}^{H}\left(x_{n}, y_{n}\right)=\varphi\left(x_{n+1}, y_{n}\right)-\varphi\left(x_{n}, y_{n}\right) .
\end{aligned}
$$

The Fried model is slightly more elaborate. The signals are computed as:

$$
\begin{aligned}
& S_{x}^{F}\left(x_{n}, y_{n}\right)=\frac{1}{2}\left\{\left[\varphi\left(x_{n}, y_{n+1}\right)-\varphi\left(x_{n}, y_{n}\right)\right]+\left[\varphi\left(x_{n+1}, y_{n+1}\right)-\varphi\left(x_{n+1}, y_{n}\right)\right]\right\} \\
& S_{y}^{F}\left(x_{n}, y_{n}\right)=\frac{1}{2}\left\{\left[\varphi\left(x_{n+1}, y_{n}\right)-\varphi\left(x_{n}, y_{n}\right)\right]+\left[\varphi\left(x_{n+1}, y_{n+1}\right)-\varphi\left(x_{n}, y_{n+1}\right)\right]\right\}
\end{aligned}
$$

In order to improve the matching between the signal model and the PWFS signals we have calibrated:

1. The wavefront sensor gain.

2. The horizontal (in $x$ ) and vertical (in $y$ ) shifts applied to the PWFS signals.

The wavefront sensor gain is a scaling factor applied to the PWFS signals in order to better match the model's signal amplitude. For the PWFS, this gain depends on the amplitude of the modulation, and in general it may be different for each subaperture. More details on these calibrations can be found in [5].

The fractional $x$ - and $y$ - shifts $\left(\Delta_{x}, \Delta_{y}\right)$ applied to the PWFS signals can be tuned to increase the correlation between the PWFS signals and the WFS model. Regarding the Hudgin model, we have found that the optimal shifts to be applied to the PWFS x-signals are $\left(\Delta_{x}, \Delta_{y}\right)=(0,0.5)$, whereas the optimal shifts to be applied to the PWFS y-signals are $\left(\Delta_{x}, \Delta_{y}\right)=(0.5,0)$ [5]. On the other hand, no shifts are required when using the Fried model. We should mention that the same optimal shifts have been found for the Shack-Hartmann wavefront sensor [6]. The inverse filter based on the Hudgin model with the optimal shifts is referred as Modified Hudgin filter (ModHud).

\section{Open-loop Reconstruction}

We will present in this section preliminary simulation results showing that it is possible to reconstruct the input wavefront using the FTR with the pyramid. Briefly, the steps required to reconstruct the wavefront are the following. The pyramid signals need to be extended in order to guarantee spatial periodicity. The simple extension methods have been used. For the Hudgin model, the simple extension method takes into account the "three-slopes" subapertures. For the Fried model, no signal recombination is performed. The resultant signals are Fourier-transformed, and the corresponding inverse filter is applied. Then, the deformable mirror compensation is applied. Finally, taking the inverse Fourier transform produces the commands vector that generates the reconstructed phase $\varphi_{\text {rec }}(\mathbf{r})$.

We will present below the evaluation of the open-loop reconstruction using the Modifed Hudgin filter. The end-to-end simulation characteristics and parameters are:

Telescope. $D=8 \mathrm{~m}$ diameter, no central obscuration.

Turbulence. Simulated with a series of independent phase realizations $\varphi_{t u r}(\mathbf{r})$ following the VonKarman model for a seeing of 0.7 " $\left(r_{0}=23.4 \mathrm{~cm} @ 750 \mathrm{~nm}\right)$, and an outer scale of $L_{0}=22 \mathrm{~m}$.

Pyramid sensor. The PWFS was simulated using an end-to-end diffractive model. The sensor parameters are:

- Sampling with 40x40 subapertures (giving a total of 1264 valid subapertures within the pupil).

- Wavefront sensing wavelength: $\lambda_{s}=750 \mathrm{~nm}$.

- Tilt modulation applied. Since the PWFS works in open loop, the amplitude of the tilt modulation needs to be of the order of $\lambda_{s} / r_{0}$ in order to avoid PWFS signal saturation.

- No measurement noise taken into account. 


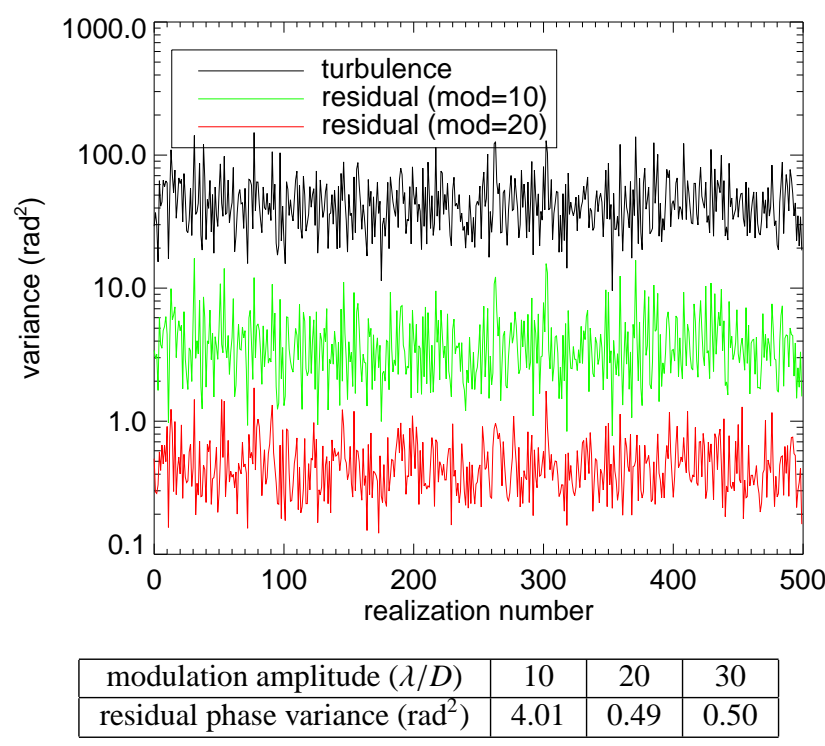

Fig. 1. Performance evaluation of the FTR in open-loop with the pyramid wavefront sensor using the Modified Hudgin filter.

Deformable mirror. 41x41 actuators (1346 valid actuators within the pupil). Bilinear influence functions with zero coupling. As a consequence, no DM compensation was required in this case.

The quality of the reconstruction can be evaluated with the variance $\sigma_{r e s}^{2}$ of the residual phase $\varphi_{\text {res }}(\mathbf{r})=\varphi_{\text {tur }}(\mathbf{r})-\varphi_{\text {rec }}(\mathbf{r})$. Figure 1 shows the variance of $\varphi_{\text {tur }}(\mathbf{r})$ and $\varphi_{\text {res }}(\mathbf{r})$ for 500 independent turbulent realizations. Three different tip-tilt modulation amplitudes were considered: 10, 20 and $30 \lambda / D$. The best performance is obtained for a modulation higher than $20 \lambda / D$. Note that the performance obtained with modulations of 20 and $30 \lambda / D$ does not change, but this is due to the absence of measurement noise in these simulations.

The residual variance of $0.5 \mathrm{rad}^{2}$ at the wavefront sensing wavelength $(750 \mathrm{~nm})$ is equivalent to a SR of $94 \%$ in $\mathrm{K}$ band. The open-loop simulations presented in this section show that a good wavefront reconstruction can be achieved using the FTR with the pyramid sensor.

\section{Closed-loop Simulations}

Let us now evaluate the performance of the FTR in closed loop. First, we will optimize the tilt modulation to match the closed-loop operating conditions. Then, we will evaluate the performance of the different reconstructors as a function of the flux level under the presence of photon noise. We will also compare the performance of the FT reconstructors with the classical matrix-vector-multiply approach. The parameters considered in the following simulations are summarized below:

Telescope. $8 \mathrm{~m}$ diameter, with a central obscuration of $14 \%$ in diameter.

Turbulence. Von-Karman turbulence with a seeing of $0.8 ”, L_{0}=40 \mathrm{~m}$, and a wind speed of $15 \mathrm{~m} / \mathrm{s}$ evolving according to the Taylor hypothesis.

Pyramid sensor. Same parameters as in the open-loop simulations presented above (40x40 subaps).

Deformable mirror. Grid of $41 \times 41$ actuators. Bicubic influence functions with a $20 \%$ of coupling were simulated. DM compensation was applied accordingly.

Temporal controller. Simple integrator with a gain of 0.8 . The sampling frequency was fixed to $1 \mathrm{kHz}$. A total delay equal to 2 frames was considered. 

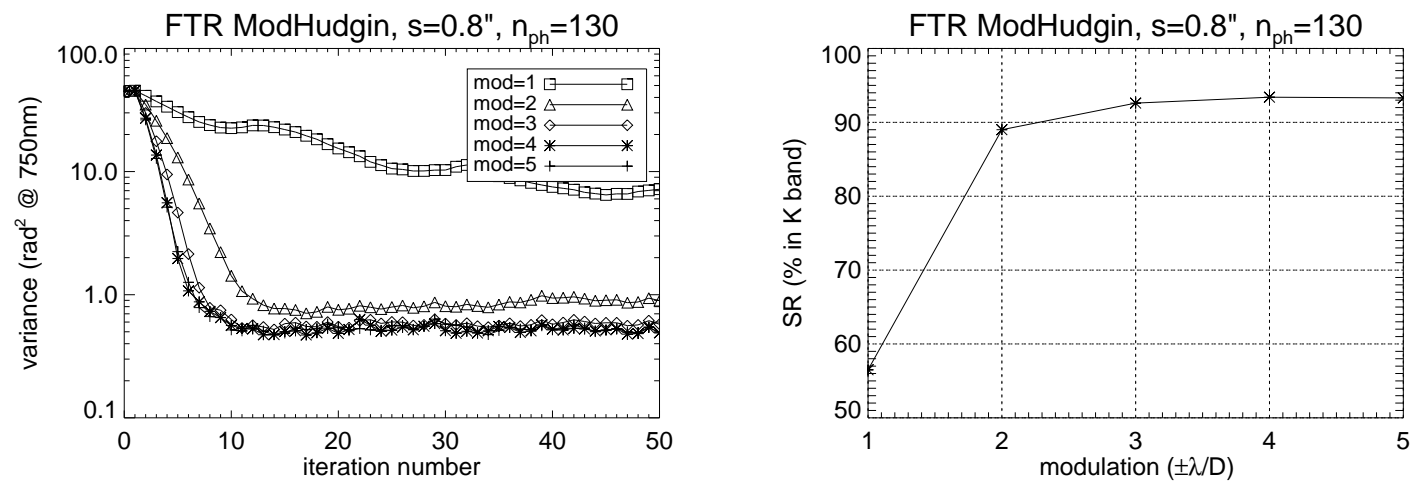

Fig. 2. Performance of the Modified Hudgin filter for different tilt modulations. (Left) Residual phase variance in $\mathrm{rad}^{2} @ \lambda_{s}=750 \mathrm{~nm}$. (Right) Strehl ratio in K band.
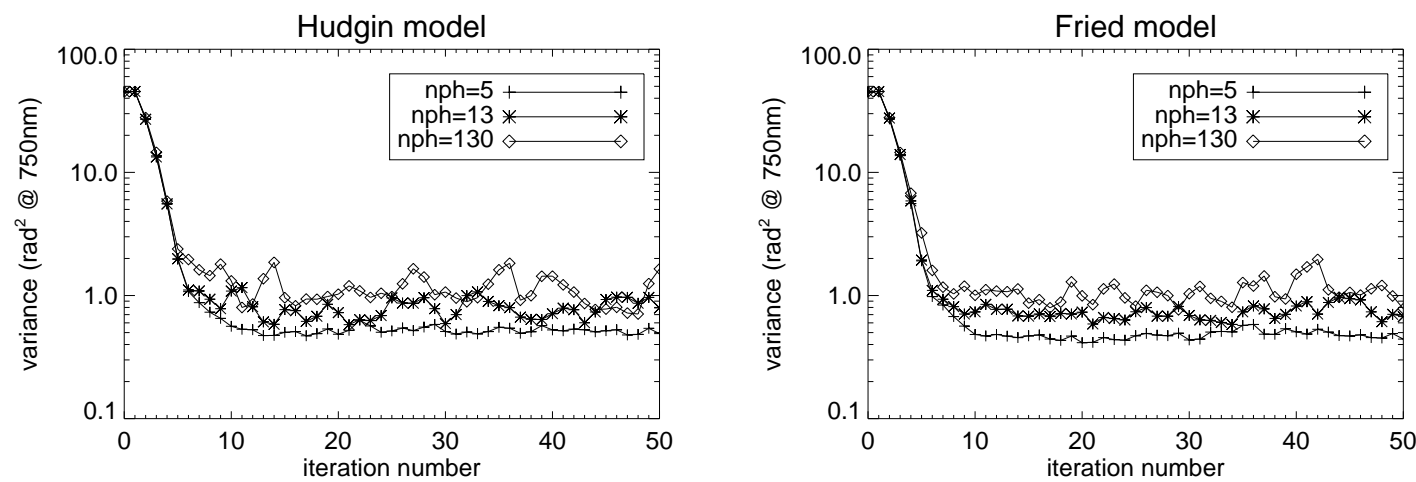

Fig. 3. Residual phase variance $\left(\operatorname{rad}^{2} @ \lambda_{s}=750 \mathrm{~nm}\right)$ at different flux levels for the first 50 loop iterations. The modulation was set to $4 \lambda / D$. (Left) Modified Hudgin filter. (Right) Fried filter.

\subsection{Effect of tilt modulation}

The tilt modulation of the pyramid wavefront sensor can be tuned to optimize the performance under the observing conditions. Let us consider a flux of $n_{p h}=130$ photons/subaperture/frame. Figure 2 shows the performance with the Modified Hudgin filter for different tilt modulations. Note that a modulation larger than $2 \lambda / D$ is required. Indeed, as shown in Figure 2(left), the system fails to converge with a modulation fo $1 \lambda / D$. The modulation that optimizes the performance under the present simulated conditions is $4 \lambda / D$, as shown in Figure 2(right).

\subsection{Performance versus flux level}

Let us now evaluate the performance of the Fourier reconstructors as a function of the flux level. Figure 3 shows the loop convergence for both Modified Hudgin and Fried reconstructors. Figure 4 shows the final performance in SR at $\mathrm{K}$ band for all the simulated cases. In the absence of readout noise, both reconstructors provide a good performance $(\mathrm{SR}>0.85)$ down to flux levels of $n_{p h}=$ 5 photons/subap/frame. The Fried model provides a slightly better performance at higher flux levels than the Modified Hudgin. On the other hand, at lower flux levels, it is the Modified Hudgin that performs slightly better due to its lower noise propagation characteristics. 


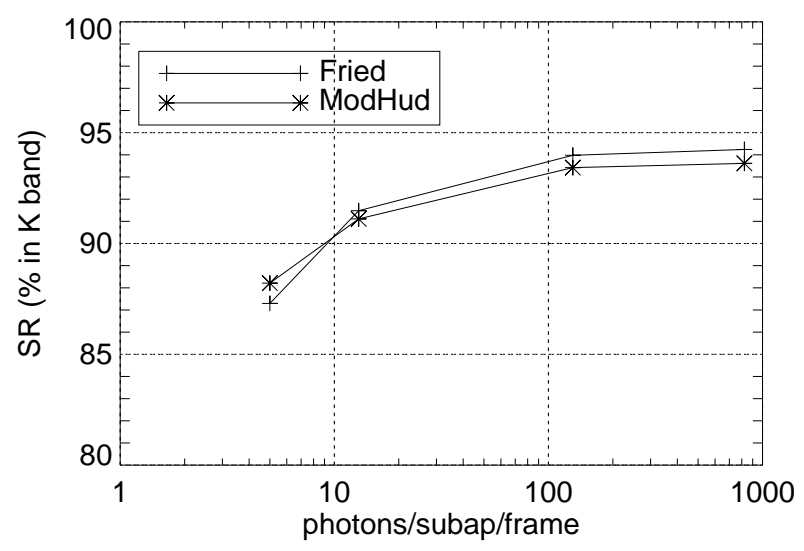

Fig. 4. Performance in Strehl ratio at $\mathrm{K}$ band as a function of the flux level obtained with the Modified Hudgin and the Fried reconstructors. The pyramid modulation was set to $4 \lambda / D$ in all cases.

\subsection{Comparison with classical reconstruction methods}

We will compare in this section the performance obtained with the Fourier and the classical reconstruction methods. The classical reconstruction approach is based on the calibration of an interaction matrix that is inverted in order to generate the reconstruction matrix. The commands vector is computed with a matrix-vector multiplication involving this reconstruction matrix and the measurement vector. This technique is also known as matrix-vector-multiply (MVM).

We have calibrated a modal interaction matrix based on $1275 \mathrm{KL}$ modes fitted by the influence functions. The modes are further re-orthonormalized on the telescope pupil. This approach to build the modal basis has been validated experimentally on the High-Order Testbench [7].

Figure 5 summarizes the results of the comparison. The flux level was set to $n_{p h}=130$ photons/subap/frame. As before, the pyramid modulation was fixed to $4 \lambda / D$ in all cases. In terms of Strehl ratio, all reconstructors give equivalent results, the MVM performing slightly better than the Fourier methods. It is interesting to analyze the modal distribution of the residual phase after correction. In order to do this, we have projected the turbulence and the residual phases onto a set of 1953 pure KL modes (radial order 61). The modal variance distribution in $\operatorname{rad}^{2} @ \lambda_{s}=750 \mathrm{~nm}$ is also shown in Figure 5. Note that the MVM provides a higher attenuation for all modes, in particular for the lowest (modes $<20)$ and highest (modes $>200)$ modes.

\section{Conclusion and Further Work}

The application of Fourier wavefront reconstruction techniques to the pyramid wavefront sensor has been demonstrated with numerical simulations. We have shown that the Modified Hudgin and the Fried inverse filters provide a similar performance in terms of SR to the classical MVM techniques. Nevertheless, a modal analysis of the correction has shown that the Fourier approach provides a smaller attenuation for all modes. This modal loss in performance of the FTR with respect to the classical MVM approach can be associated to the fact that the signal models used (Hudgin and Fried) are simplified representations of the pyramid signals.

The optimization of the modal performance attained by the FTR needs to be further investigated. The loss in performance may not be negligible in the case of the XAO systems currently under investigation for the ELTs, comprising $\approx 30000$ subapertures and actuators [1]. As a future work, we will focus on the derivation of an ad-hoc inverse filter for the pyramid sensor based on the diffractive models of the signals. The experimental validation of the Fourier reconstruction techniques for 


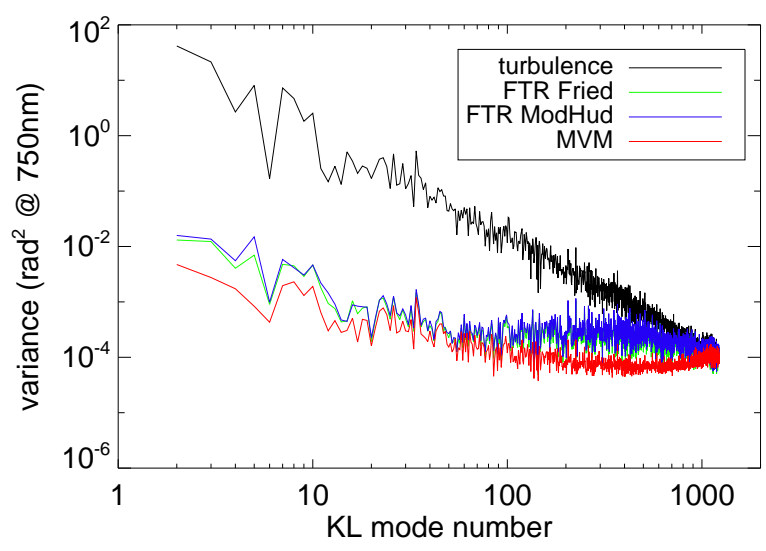

\begin{tabular}{|c|c|c|c|}
\hline & MVM & FTR (Fried) & FTR (ModHud) \\
\hline SR (\% in K) & 96 & 94 & 93 \\
\hline
\end{tabular}

Fig. 5. Performance comparison between the matrix-vector multiply (MVM) and the Fourier reconstruction methods.

the pyramid wavefront sensor may also be carried out in the near future on the High-order Testbench (HOT) currently installed at ESO Garching.

\section{References}

1. C. Vérinaud, R. Mollard, P. Kern, R. Galicher, P. Baudoz, J.-L. Beuzit, and Gérard Rousset. Postcoronagraphic wave-front sensing for the exo-planet imaging camera and spectrograph, EPICS for the European ELT, in: Adaptive Optics Systems, Proc. of SPIE 7015 (2008), 70151W

2. L. A. Poyneer, D. T. Gavel, and J. M. Brase. Advanced techniques for Fourier transform wavefront reconstruction, JOSAA 4839 (2002), 2100-2111

3. S. Esposito, O. Feeney, and A. Riccardi. Laboratory test of a pyramid wavefront sensor, in: Adaptive Optical Systems Technology, Proc. of SPIE 4007 (2000), 416-422

4. A. Burvall, E. Daly, S. R. Chamot, and C. Dainty. Linearity of the pyramid wavefront sensor, Optics Express 14 (2006), 11925-11934

5. F. Quirós-Pacheco, C. Correia, and S. Esposito. ELT Design Study, WP9600 Technical Report ELT-TRE-INA-09600-0019, July 2009.

6. L. A. Poyneer. Advanced techniques for Fourier transform wavefront reconstruction, in: Adaptive Optical Systems Technologies II, Proc. of SPIE 4839 (2002), 1023-1033

7. E. Pinna, A. T. Puglisi, F. Quirós-Pacheco, L. Busoni, A. Tozzi, S. Esposito, E. Aller-Carpentier, and M. Kasper. The pyramid wavefront sensor for the High Order Testbench (HOT), in: Adaptive Optics Systems, Proc. of SPIE 7015 (2008), 701559 